\title{
Culture institutionnelle et apprentissage en ligne en Afrique subsaharienne francophone : le cas d'une école normale supérieure camerounaise
}

\author{
Colette Mvoto Meyong \\ Université d'Ottawa, CANADA \\ mecoco61@yahoo.fr
}

Rélexion pédagogique

\section{Résumé}

La présente recherche s'inscrit dans un courant de réflexion sur les stratégies permettant à l'université d'accomplir sa mission. Elle porte un regard particulier sur les facteurs jugés favorables à l'implantation de l'apprentissage en ligne, à travers les représentations sociales (RS) des étudiants et des enseignants de l'École Normale Supérieure d'Enseignement Technique (ENSET) du Cameroun. L'étude comprend quatre sections: la première traite de la problématique et pose une première question de recherche; la seconde est un cadre de référence qui décrit les concepts clés et adapte des modèles de compréhension afin de formuler des questions spécifiques, rattachées à la première; la troisième justifie l'orientation épistémologique de l'analyse et les choix méthodologiques du chercheur. Finalement, la quatrième section présente l'analyse ethnographique du contenu discursif reconstitué et met en exergue la contribution de ses résultats. La conclusion souligne les limites et implications de la recherche.

\section{Summary}

This study is part of an ongoing concern with the strategies that allow the university to achieve its goals. It focuses on the factors that promote implementation of online learning, by examing the social representations (SR) of students and teachers at the École Normale Supérieure d'Enseignement Technique (ENSET) in Cameroon. The study includes four sections : the first examines the problem and poses a main research question; the second presents a theoretical framework which describes the key concepts in play and shows how existing models can be adapted in order to formulate specific subquestions that relate to the main question; the third section justifies the epistemological stance taken for the analysis and the methodological choices made by the researcher. Finally, the fourth section presents the ethnographic content analysis of the discourse samples as they were reconstructed, and pinpoints the contribution made by the results. A short conclusion underlines the limits of the study and implications for future research. 


\section{Problématique}

La structure sociogéographique du continent africain permet de distinguer le Maghreb (Afrique nordsahariennne) de l'Afrique subsaharienne (ASS), qui se subdivise en trois groupes de pays: l'ASS francophone (ASSF), l'ASS lusophone, et l'ASS anglophone (Jessen, 1999, p. 203). Nées au lendemain de la Deuxième Guerre mondiale, les universités d'ASSF devaient répondre aux aspirations des populations affranchies du joug colonial (Assié-Lumumba, 1993; Samoff, AssiéLumumba, Jallade et Cohen, 1996). Quelques pays disposaient d'un seul campus universitaire, comme ce fut le cas du Cameroun avec l'Université fédérale de Yaoundé (Ministère de l'Enseignement Supérieur du Cameroun, 2001, 2004) Les impératifs de croissance et les besoins de spécialisation imposent à ce pays une nécessaire création continue de nouvelles institutions d'enseignement supérieur. Ce faisant, les six universités d'État locales subissent les effets d'un environnement engagé dans les échanges mondiaux complémentaires et compétitifs, tant leurs besoins criants et multidimensionnels (Ékome Engolo, 2000) émergent des vestiges du passé et des blocages endogènes et exogènes (Butcher, 2004; Kom, 2000).

Ces besoins affectent la volonté de former des femmes et des hommes créateurs et initiateurs, capables d'affronter les problèmes contextuels. Dans cette perspective d'accomplissement d'une «mission historique d'enseignement, de recherche et de développement communautaire, [les universités camerounaises] remettent en cause les procédures établies, en concevant des modèles qui marchent» (Bollag, 2004, p. 33). Des obstacles d'ordre économique, politique, et culturel ressources matérielles, censure et favoritisme, traditions et croyances, idéologie et philosophie éducative - (Saint, 1999; Seck, 1997) alourdissent cette mission. Le modèle organisationnel dominant met en évidence une gestion administrative qui freine l'évolution des méthodes pédagogiques
(Affa'a, Grisé et Verna, 2003): un problème de formation continue et de maintien du corps enseignant se pose (Bekele, 2001; Gauthier, Bissonnette, Richard et Djibo, 2003).

Dans cet état des lieux, nous abordons le cas de l'École normale supérieure d'enseignement technique (ENSET) du Cameroun en considérant des problèmes d'ordre culturel, et ceux qui sont reliés au manque de ressources matérielles (désuétude des infrastructures d'accueil, pauvreté des laboratoires et bibliothèques, besoin de technologies de l'information et de la communication [TIC], etc.). Cette école est aux prises avec d'autres phénomènes alarmants - crise financière quasi chronique, hétérogénéité des niveaux de formation, effectifs pléthoriques, insuffisance $\mathrm{du}$ personnel d'encadrement, etc. (Fonkoua, 2006; Tcheeko, Yatchou et Tangha, 2001). Comme remède à ces problèmes, l'exploitation pédagogique de la communication médiatisée par ordinateur (CMO) s'impose.

Tenue pour acquise pour ce qui est de l'amélioration de la qualité de la formation universitaire (Sauvé, Wright et St-Pierre, 2004), cette exploitation peut, au moins, permettre de surmonter les problèmes d'hétérogénéité des niveaux et des effectifs pléthoriques, et donner une grande liberté d'action à l'étudiant : aucun exposé professoral ne pourrait le faire. Cependant, il faut, d'une part, noter que même dans les pays industrialisés, certains enseignants affichent encore une indifférence à l'exploitation pédagogique des TIC (Charlier, 2000; Desjardins, 2005; Karsenti, Savoie-Zajc et Larose, 2001) et, d'autre part, reconnaître l'impact des conditions sociohistoriques et matérielles contextuelles (Debeb, 2001; Loiret, 2005; Murphy, Anzalone, Bosch et Moulton, 2002).

Sous cet angle, l'histoire rappelle que la première université camerounaise fut un atout politique. Isolé de son environnement social, à l'image d'un monastère se pointant comme une tour d'ivoire, ce 
temple du savoir était réservé à une poignée d'élus à initier aux rites de la Science et des belles-lettres de Molière. Le respect de l'autorité, du droit d'aînesse et des lois religieuses contribue au maintien de méthodes pédagogiques rigides, défavorables au partage des connaissances (Ng'ethe, 2003). Mais une exploitation efficiente des TIC dans les universités ne va pas de soi : elle exige une implication politique et institutionnelle, dans une obligation de résultat. Elle n'est pas non plus nouvelle, car au Cameroun, chaque génération de technologies s'accompagne d'expériences pédagogiques plus ou moins menées à terme (Essono, 2003; Valérien, Guidon, Wallet et Brunswic, 2003).

Comme le présument Leborgne-Tahiri (2002) et Ng'ethe (2003), dans les universités ASSF, l'enseignant qui s'interroge sur son nouveau rôle peut craindre de devenir un simple répétiteur et devenir impatient quant aux retombées de l'exploitation pédagogique de la CMO. Pour sa part, l'apprenant, habitué à communiquer face à face avec son professeur, peut penser que la relation pédagogique virtuelle manque d'affectivité. Bien que divergentes selon qu'on est professeur ou étudiant, ces appréhensions peuvent être concomitantes quant à l'accomplissement de la mission essentielle de l'ENSET, compte tenu des obstacles contextuels susmentionnés et des contraintes associées aux avantages de l'exploitation pédagogique de la $\mathrm{CMO}$ (voir le Tableau 1 en annexe).

Face aux contraintes qu'introduit la CMO dans la situation de formation à l'étude, ses acteurs risquent de demeurer prudents et hésitants quant à l'implantation de l'apprentissage en ligne. Il s'agit donc de trouver les mécanismes pouvant rendre cette entreprise moins réfractaire, en tenant compte de leurs RS, parfois divergentes: quelles sont les représentations sociales dominantes chez les étudiants et les enseignants de l'ENSET quant aux facteurs jugés favorables au changement paradigmatique de la formation sous-jacent à l'innovation pédagogique en cours dans leur école, au regard de sa culture institutionnelle? Telle est la question générale de recherche qui se pose. Un agencement d'éléments théoriques de référence conduit à deux questions spécifiques.

\section{Cadre de référence}

Afin de trouver des éléments de réponse crédibles à la principale question posée, cette section clarifie et relie les concepts clés de la recherche: RS et paradigme éducationnel, innovation et culture institutionnelle. Elle adapte aussi des modèles d'analyse à son contexte.

\subsection{Représentations sociales et paradigme éducationnel}

De la littérature (Atlet, 2001; Baillauqués, 2001; Jodelet, 1991; Moscovici, 1961; etc.) traitant de l'utilité des RS, on retient qu'elles sont des systèmes de construction (processus) des connaissances (produits) dont l'analyse facilite la compréhension de la réalité. Cette compréhension est ontologique qu'est-ce que c'est que le monde? -, épistémologique - comment le connaître? - et praxéologique - comment y agir? - (Paillé et Mucchielli, 2003, p. 43). Des recherches interprétatives des RS (Boissonneault, 2002; Marchand et Loisier, 2003; Savoie-Zajc, 2001) réalisées au cœur même des institutions universitaires confirment que ces productions cognitives orientent les jugements et les choix d'une nouvelle manière commune de penser et, implicitement, celui des pratiques pédagogiques transigeant avec l'exploitation de la CMO.

Ces choix symbolisent l'émergence d'un nouveau paradigme, une vision $\mathrm{du}$ monde guidée par un ensemble de règles écrites ou tacites indiquant comment s'organiser naturellement face aux événements (Cartier, 2001; Marchand, 1998, 2001). Autrement dit, un paradigme est ce protocole de conceptions qui détermine, entre autres, la distance réflexive que prennent les acteurs d'une situation de 
formation par rapport aux nouvelles pratiques professionnelles. Il donne naissance à un paradigme secondaire, dit "éducationnel», un régulateur des généralisations symboliques (RS, croyances, valeurs, etc.) et des techniques valorisées (formation, recherche, gestion administrative, etc.), dites « exemplaires» (Bertrand et Valois, 1999). Ainsi, le paradigme éducationnel détermine l'adoption d'un changement, d'une innovation ou d'une nouvelle culture institutionnelle.

\subsection{Changement, innovation pédagogique et culture institutionnelle}

Comme tout changement, une innovation améliore les techniques exemplaires (Savoie-Zajc, 1993). Dans une situation de formation, en introduisant un nouvel élément, l'implantation d'une innovation pédagogique s'observe par son évolution d'un état perçu comme étant meilleur (Adamczewzki, 1996; Lapointe, 1996). Cette implantation consiste à annuler la différence $\left(t_{n+1}-t_{n}\right)$ si et seulement si les

parties prenantes modifient leurs RS et, par ricochet, abandonnent une vieille culture institutionnelle pour s'accommoder à une autre.

Soit dit en passant, le mot «culture» signifie prendre soin de la terre, à la façon des agriculteurs, ou prendre soin des dieux, dans la pratique du culte (Hans-Drouin, 1996, p. 2) : il intègre des activités physiques et spirituelles de tout être humain, condamné à combler ses besoins. La notion de culture institutionnelle désigne, quant à elle, un ensemble de productions et de significations auxquelles s'identifient ou s'opposent des acteurs d'une institution. Elle est l'unité de changement la plus complexe d'une situation de formation (SavoieZajc et Dolbec, 1996), comme celle de l'ENSET. $t_{n}$, jugé insatisfaisant, vers un état souhaité $t_{n+1}$,

Cette culture collective teinte leurs RS (Charlier, Bonamy et Saunders, 2003; Docq et Daele, 2003). Ainsi, créer un changement, c'est agir sur une culture (Brénot et Tuvée, 1996, p. 28). S'agissant de l'implantation de l'apprentissage en ligne, ce changement est d'ordre paradigmatique : une mutation conceptuelle de la formation (Linard, 2003), du « processus enseigner» vers le «processus apprendre» (Houssaye, 1988, p. 41). Pour cerner la portée de ce propos, il faut adapter des modèles d'analyse au contexte de la recherche.

\subsection{Modèles d'analyse adaptés au contexte de la recherche}

En référence à la proposition d'implantation d'une innovation (voir la Figure 1) de Collerette et Delisle (1982, p. 66), la présente étude correspond à sa première étape: le diagnostic de la situation problématique.

Figure 1. Modèle Implantation d'une innovation (adapté de Collerette et Delisle, 1982, p. 66)

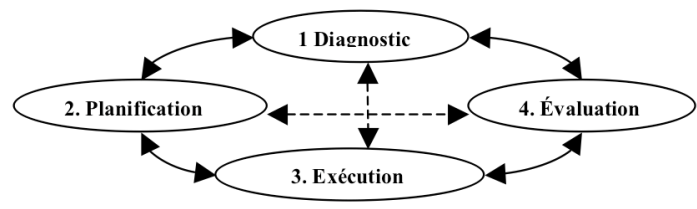

$\mathrm{Au}$ sujet $\mathrm{du}$ diagnostic d'une situation insatisfaisante, Collerette et Delisle (voir la figure 1) conseillent de recueillir des informations en consultant les acteurs de terrain et de les transformer en données permettant de déceler des facteurs qui influent sur la planification du changement. Dans une dynamique rhétorique, à l'image de leur modèle, l'analyse des RS sur le plan de la conjoncture générale d'une mutation paradigmatique de la formation par le truchement

Figure 2. Modèle systémique SOCA d'une situation pédagogique en ligne

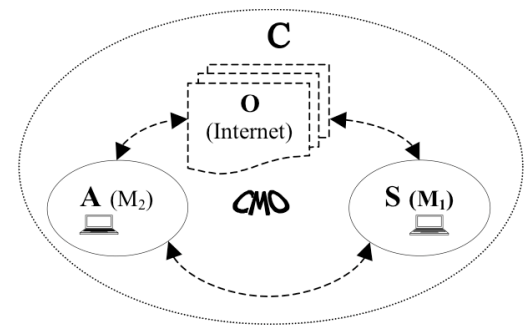
www.profetic.org/revue 
de la CMO se réfère au modèle systémique SOCA (voir la Figure 2).

Le modèle SOCA d'une situation d'apprentissage s'inspire du modèle SOMA de Legendre (1983, p. 277). Selon Legendre, le Sujet $S$ désigne l'apprenant (individu, ou groupe); l'Objet $\boldsymbol{O}$ englobe les documents administratifs et les contenus d'apprentissage; le Milieu $\boldsymbol{M}$ correspond à l'environnement pédagogique et l'Agent $A$ désigne toutes les ressources humaines et matérielles exploitables par $S$. Dans le modèle SOCA, la description de $\mathbf{S}$ est identique à celle de Legendre; $\mathbf{O}$ englobe les contenus pédagogiques en ligne à choisir, déconstruire et reconstituer; la lettre C désigne une communauté virtuelle d'apprentissage (personnes et dispositifs techniques de médiatisation et de médiation); et l'Accompagnateur A est toute personne facilitatrice de l'apprentissage. L'efficacité d'un tel accompagnement dépend des compétences de $\mathbf{A}$ et de $\mathbf{S}$, et des caractéristiques de $\mathbf{M}_{1}$ et $\mathbf{M}_{2}$, dont les différences ajoutent à la diversité culturelle de $\mathbf{C}$, une source potentielle d'obstacles à l'exploitation de la CMO (Perriault, 2002; Trégouët, 1998).

Inhiber ces obstacles signifie, entre autres, toujours développer des compétences pédagogiques transversales (Desjardins, 2005; Prayal et Gignac, 2004) et muter une culture institutionnelle en s'accommodant de celle de C (Brunet, 2001). Une lecture avancée des écrits consultés permet d'affirmer que l'on comprend mieux comment garantir le succès de ces actions à travers une étude des RS coproduites, au plus proche du naturel. Deux questions spécifiques de recherche guident ce travail d'analyse : a) Comment les étudiants et les enseignants de l'ENSET se représentent-ils la situation de formation de leur école, en matière de culture institutionnelle? b) Comment ces acteurs se représentent-ils la situation de formation souhaitée, en rapport avec le succès de l'exploitation pédagogique de la CMO? Une démarche qualitative interprétative $\mathrm{s}$ 'avère pertinente pour répondre à ces questions.

\section{Orientation épistémologique de l'analyse et choix méthodologiques du chercheur}

Analyser des RS en tant que systèmes de connaissances pour cerner les conditions d'innovation de l'objet abstrait qu'est une situation de formation nécessite l'adoption d'une démarche qualitative interprétative. Eu égard à la réalité spécifique de l'ENSET, le chercheur s'éloigne des généralisations et valorise plutôt la singularité. La carence de recherches empiriques de cette envergure dans le contexte investigué octroie à la présente étude descriptive une nature exploratoire. Pour rester ouverte et intersubjective, elle s'appuie sur l'approche systémique.

Afin de repérer des points de convergence et de divergence des RS recueillies, le chercheur reste sensible au discours argumentatif et observe les principes de l'analyse ethnographique, dans la perspective de l'ethnométhodologie (Garfinkel, 1967, 2001; Coulon, 1996). Cette variante de la microsociologie postule que l'interprétation de la réalité est tributaire des constructions de sens émergeant des interactions personnelles et rattachées à des événements concrets. En consignant de tenir compte de la dimension naturelle des participants et des implications de leur intersubjectivité, ses principes corroborent ceux de la recherche qualitative interprétative (Savoie-Zajc, 2000). En ce sens, la présente étude de cas reconstitue les RS coproduites sur le terrain, sans les déformer, une façon de répondre aux questions (Paillé et Mucchielli, 2003, p. 109; Van Der Maren, 1999, p. 198) que se posent les répondants face au changement vécu.

Ce faisant, le chercheur utilise trois techniques de terrain : a) le questionnaire d'enquête écrit, qui lui permet d'identifier 70 sujets consentants (54 étudiants en fin de formation et 16 enseignants); b) le journal de bord, dans lequel il note les détails importants du déroulement de la recherche; et c) 
l'entrevue de groupe, principal moyen de collecte des RS. Cette collecte des données se déroule dans l'enceinte de l'ENSET en deux temps : a) de janvier à mai 2003 (prise de contact, clarification des objectifs de l'étude, obtention du consentement, sélection et regroupement des interviewés); et b) en janvier 2004 (présentation de la reconstitution des données aux enseignants pour confirmation).

Lors de la première descente sur le terrain, selon la disponibilité des répondants, le chercheur constitue cinq groupes d'étudiants de cinq sujets consentants $(25 / 54)$ choisis au hasard simple, et un groupe de cinq enseignants, approchés à la sortie d'une réunion de travail. Cependant, un échantillonnage par saturation de la connaissance ${ }^{1}$ limite le nombre de groupes de discussion avec les étudiants à trois (15 sujets au total). Pour ce faire, la transcription progressive des entretiens sur le terrain amorce l'analyse des RS reconstituées. À la suite d'une première analyse de ces données, afin de compléter les suggestions quant à l'exploitation pédagogique de la CMO, en janvier 2004, le chercheur s'entretient avec un second groupe de cinq enseignants consentants ayant pris connaissance des RS reconstituées.

\section{Reconstitution et analyse des RS}

À titre de rappel, l'analyse qualitative interprétative des RS recueillies veut guider la connaissance des possibilités d'amélioration de la qualité de la formation dans les universités d'ASSF et la compréhension du comment accélérer et maintenir le changement paradigmatique de la formation par l'exploitation de la CMO. Le choix du modèle mixte d'analyse de contenu permet au chercheur de considérer à la fois les catégories prédéterminées et celles qui émergent des verbatim (Paillé et Mucchielli, 2003). Ce faisant, il classe les unités de sens retenues sous trois thèmes : a) RS face à la situation de formation vécue; b) RS quant à la situation de formation souhaitée; et c) RS quant à l'exploitation pédagogique de la $C M O$, au regard de l'état des lieux étudiés.

Après le codage des verbatim, le chercheur retient une unité de sens lorsque sa fréquence d'apparition est significative, ou si elle présente une originalité par rapport aux écrits consultés. Afin de déceler les points de divergence et de convergence de RS formulées sous une catégorie thématique, il compare progressivement les significations qu'il en donne. Hormis quelques divergences justifiables par la différence des statuts (étudiant ou professeur) des membres des groupes de discussion, la synthèse de notre compréhension interprétative se développe par thème.

Sous le premier thème, RS face à la situation de formation vécue, les répondants décrivent la culture institutionnelle de leur école dans une remise en cause des pratiques professionnelles les plus courantes. Sans cesser de rappeler l'ampleur de leurs besoins, ils émettent clairement des doutes sur la bonne qualité des méthodes de la gestion administrative (planification et collaboration) et pédagogique (enseignement et évaluation). Ils confirment ainsi les obstacles contextuels à l'amélioration des prestations de services de formation relevés dans la problématique (Ékome Engolo, 2000; Gauthier et al., 2003) et les appréhensions (Leborgne-Tahiri, 2002; Ng'ethe, 2003) d'interrogations, de craintes, et d'inquiétudes des acteurs des universités d'ASSF face aux contraintes inhérentes à une exploitation pédagogique de la CMO (voir le Tableau 1 à l'Annexe 1).

Sous le second thème, RS quant à la situation de formation souhaitée, les répondants clarifient leur conception de l'acte pédagogique. Ils mettent en évidence le changement du paradigme de la formation dans lequel ils sont déjà engagés lorsqu'ils expriment globalement une pensée constructiviste de l'enseignement ${ }^{2}$ (Lebrun, 2002; Martel, 2002). Par ordre de priorité, ils souhaitent: 
a) la valorisation de la profession enseignante et une autonomisation des administrateurs, de la part des décideurs politiques; b) l'élaboration d'un code d'éthique concernant tous les acteurs dans les prises de décision; c) la formation continue du personnel d'encadrement; d) la rénovation des infrastructures existantes, avant même de construire des laboratoires modernes, afin de les adapter à l'exploitation pédagogique de la CMO.

Sous le troisième thème, notre reconstitution interprétative des $R S$ quant à l'exploitation pédagogique de la CMO révèle que tous les répondants sont conscients des contraintes à surmonter pour une accommodation culturelle et collective à la dynamique d'une communauté virtuelle d'apprentissage. En plus des souhaits exprimés sous le second thème, les répondants suggèrent d'entreprendre, au préalable, une étude de faisabilité incluant l'évaluation des coûts des ressources nécessaires pour implanter un système de formation en ligne dans leur école. Ils insistent aussi sur la réalisation d'une analyse profonde de
Les enseignants rappellent que la recherche participative influence positivement la qualité des méthodes de gestion administrative: elle permet d'engager toute l'institution dans le changement. Selon eux, adopter cette stratégie de collaboration dans la résolution de leurs problèmes mettrait en valeur la diversité des compétences que l'on retrouve à l'ENSET, en tant qu'école de formation à l'enseignement technique. Cette possibilité d'améliorer la qualité des rapports et des pratiques professionnelles peut, à leur avis, accélérer l'abandon d'une culture institutionnelle héritée de la colonisation, que dénoncent particulièrement les étudiants.

Soit dit en passant, comme les enseignants, qui citent les facteurs garants de l'abandon de cette culture, les étudiants reviennent sans cesse sur les contraintes de la $\mathrm{CMO}$, et notamment, sur l'obligation: a) de remettre en question des conceptions traditionnelles de leurs rôles sur une scène pédagogique; b) de disposer chacun d'un ordinateur de pointe; c) d'entretenir les laboratoires

Figure 3. Modèle d'insertion pédagogique de la CMO dans une situation conventionnelle de formation face à face

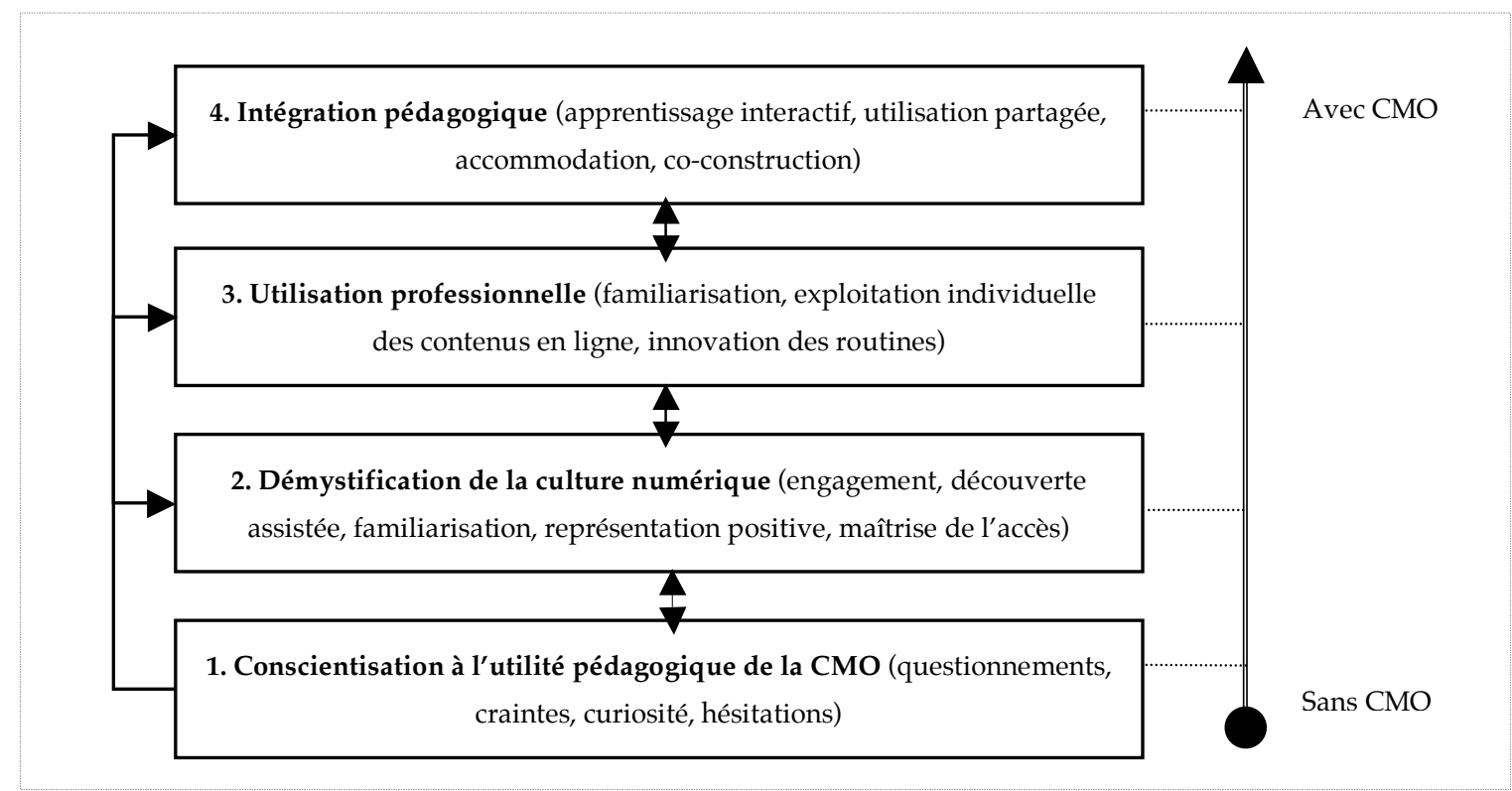

leurs besoins, sous forme de recherche participative. et équipements informatiques; et d) de payer les droits d'accès au réseau Internet et à certains 
contenus électroniques. Finalement, en reconstituant les suggestions recensées, on comprend que, selon les répondants, sensibiliser en motivant est indispensable pour démystifier la complexité de l'apprentissage en ligne et mieux préparer les adhérents à surmonter ses contraintes. Il en découle un modèle d'insertion pédagogique de la CMO dans une situation conventionnelle de formation universitaire (voir la Figure 3).

Le modèle élaboré illustre un processus non linéaire en quatre étapes, que nous situons sur un continuum allant d'un enseignement conventionnel de type magistral (sans $\mathrm{CMO}$ ) à un apprentissage à distance ou interactif en ligne (avec CMO). À l'étape initiale, sensibilisation à l'utilité pédagogique de la $C M O$, perturbé et habité par un mélange d'inquiétudes, de craintes, d'hésitations et de curiosité, le sujet connaissant se questionne quant à ses compétences d'ordre technologique et son rôle sur une scène de formation en ligne. À la seconde, démystification de la culture numérique, assisté par un accompagnateur, il commence à se familiariser avec la nouveauté et développe des habiletés d'accès aux contenus en ligne. À la troisième, utilisation professionnelle, tout en continuant à s'accommoder à la culture numérique, il parvient déjà à utiliser de façon autonome les outils et contenus d'Internet. C'est à la quatrième étape, intégration pédagogique de

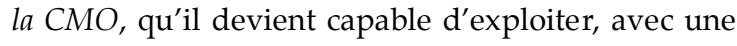
certaine assurance, cet outil d'apprentissage interactif.

\section{Conclusion}

L'exploitation pédagogique de la CMO est encore en expérimentation dans la plupart des universités d'ASSF et, cette analyse étant une première dans son contexte de réalisation, sa nature qualitative, exploratoire et descriptive met en exergue les pistes de recherche ouvertes. Même si son approche méthodologique est transférable, le petit nombre de participants et les limites inhérentes à toute étude qualitative (choix intentionnel, subjectivité du chercheur, contingences du terrain, etc.) rendent ses résultats non généralisables.

\section{Références}

Adamczewki, G. (1996). La notion d'innovation : figures majeures et métaphores oubliées. Dans F. Cros et G. Adamczewki (dir.), L'innovation en éducation et en formation (p. 15-30). Bruxelles : De Boeck Université.

Affa'a, F.-M., Grisé, J. et Verna, G. (2003). À propos de quelques obstacles à la mise en œuvre du plan stratégique de développement de l'enseignement supérieur $d u$ Cameroun. Document de travail 2003-039. Québec: Faculté des sciences de l'administration de l’Université Laval. Récupéré le 5 février 2007 du site de la faculté, section Recherche - Publications Documents de travail - Série électronique 2003: http :/ / rd.fsa.ulaval.ca/v2/ctr_doc/documents/ 2003-039.pdf

Assié-Lumumba, T. (1993). L'enseignement supérieur en Afrique francophone: évaluation $d u$ potentiel des universités classiques et des alternatives pour le développement. Washington, DC : Banque Mondiale.

Altet, M. (2001). Les compétences de l'enseignantprofessionnel : entre savoirs, schèmes d'action et adaptation, le savoir analysé. Dans L. Paquay, M. Altet, E. Charlier et P. Perrenoud (dir.), Former des

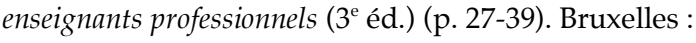
De Boeck Université.

Baillauqués, S. (2001). Le travail des représentations dans la formation des enseignants. Dans L. Paquay, M. Altet, E. Charlier et P. Perrenoud (dir.), Former des

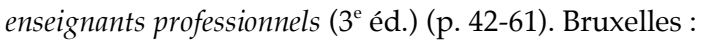
De Boeck Université**

Bekele, A. (2001). Quelques réflexions sur les universités africaines : la quête pour une maind'œuvre qualifiée. Bulletin de l'Institut international de l'UNESCO pour le renforcement des capacités en Afrique (IIRCA), 3(3), p. 2-7. Récupéré le 11 février 2007 du site de l'Institut, section Newsletter Archive : http:/ / www.unesco-iicba.org 
Bertrand, Y. et Valois, P. (1999). Fondements éducatifs pour une nouvelle société. Montréal: Éditions Nouvelles.

Boissonneault, J. (2002). Représentations de l'enseignement et de l'apprentissage par les professeurs universitaires utilisant des médias interactifs à distance. Thèse présentée à la Faculté des Études supérieures et de la recherche de l'Université d'Ottawa pour l'obtention du grade de Philosophiae Doctor (Ph. D.). Faculté d'éducation, mai 2002.

Bollag, B. (2004). Amélioration de l'enseignement supérieur en Afrique sub-saharienne: ce qui marche! Rapport d'une conférence régionale de formation tenue à Accra, Ghana. Washington, DC: Banque internationale pour la reconstruction et le développement / Banque Mondiale. Récupéré le 15 février 2007 du site de la Banque Mondiale, section Countries - Africa Development Topics - Tertiary Education in Africa - A Training Conference on Tertiary Education in Africa: http:/ / siteresources.worldbank.org/INTAFRREGT OPTEIA/Resources/final_fr.pdf

Brénot, J. et Tuvée, L. (1996). Le changement dans les organisations. Paris: Presses Universitaires de France.

Brunet, P. J. (2001, septembre). D'un nouveau mode de pensée à un modèle éthique d'intégration d'Internet. Communication présentée au Colloque Initiatives 2001: éthique et nouvelles technologies, l'appropriation des savoirs en question, tenu lors du $9^{\text {e }}$ Sommet de la Francophonie, Beyrouth, Liban. Récupéré le 12 février 2007 du site des notes du colloque, section de la Session 3: http:/ / www.initiatives.refer.org/_notes/session3. $\underline{\mathrm{htm}}$

Butcher, N. (2004, mai). L'infrastructure technologique et l'utilisation des TIC dans le secteur de l'éducation en Afrique: vue générale. Paris: Groupe de travail sur l'enseignement à distance et l'apprentissage libre de l'Association pour le développement de l'éducation en Afrique (ADEA). Récupéré le 12 février 2007 du site de l'association, section Publications - Catalogue des publications de l'ADEA - Groupe de travail sur l'enseignement à distance et l'apprentissage libre : http://www.adeanet.org/publications/fr_pubs_wg de.html

Cartier, M. (2001). Les inforoutes et l'éducation, mythes et réalités. Pour passer des infrastructures aux inforoutes. Dans M. Kaszap, D. Jeffery et G. Lemire (dir.), Exploration d'Internet, recherche en éducation et rôles des professionnels de l'enseignement (p. 9-59). Sainte-Foy, Canada: Presses de l'Université Laval; Paris: L'Harmattan.

Charlier, B. (2000). Dispositifs médiatisés, leviers d'autoformation: concepts et pratiques. Dans S. Alava (dir.), Cyberespace et formations ouvertes. Vers une mutation des pratiques de formation (p. 81-97). Bruxelles: De Boeck Université.

Charlier, B., Bonamy, J. et Saunders, M. (2003). Apprivoiser l'innovation. Dans B. Charlier et D. Peraya (dir.), Technologie et innovation en pédagogie. Dispositifs innovants pour l'enseignement supérieur (p. 43-63). Bruxelles: De Boeck Université.

Collerette, P. et Delisle, G. (dir.). (1982). Le changement planifié. Québec : Agence d'Arc.

Coulon, A. (1996). L'ethnométhodologie. Paris: Presses Universitaires de France.

Debeb, G. E. (2001). L'éducation à distance dans les universités africaines : justification, situation actuelle et perspectives. Bulletin de l'Institut international de l'UNESCO pour le renforcement des capacités en Afrique (IIRCA), 3(3), p. 15-18. Récupéré le 14 février 2007 du cite de l'Institut, section Newsletter Archive: http://www.unesco-iicba.org

Desjardins, F. (2005). Les représentations des enseignants quant à leurs profils de compétences relatives à l'ordinateur : vers une théorie des TIC en éducation. La revue canadienne de l'apprentissage et de la technologie, 32(1), p. 27-49.

Docq, F. et Daele, A. (2003). De l'outil à l'instrument : des usages en émergence. Dans B. Charlier et D. Peraya (dir.), Technologie et innovation en pédagogie. 
Dispositifs innovants pour l'enseignement supérieur (p. 113-128). Bruxelles : De Boeck Université.

Ekome Engolo, C. (2000). Les universités africaines dans le sillage de la mondialisation. Mots pluriels, numéro 32-avril 2000. Récupéré le 12 mars 2007 du site de la revue http://www.arts.uwa.edu.au/ $\underline{\text { MP1300ee.html }}$

Essono, L. M. O. (2003, août). La formation à distance en Afrique francophone à l'heure des TIC. Bilan, perspectives et interrogations. Communication présentée au colloque international Les fractures nord/sud en question. Quels enjeux? Quels partenariats? Gironde, France. Récupéré le 14 février 2007 du site du projet Africa'nti, section Résultats - Colloques organisés - L'éducation à distance: http://www.afri canti.org/resultats/colloque2003/col2003_prog.htm

Fonkoua, P. (2006). La formation des enseignants et le développement durable en Afrique : d'une situation locale à une préoccupation globale. Formation et profession. Bulletin $d u$ Centre de recherche interuniversitaire sur la formation et la profession enseignante (CRIFPE), 12(1), p. 43-49. Récupéré le 14 février $2007 \mathrm{du}$ site du bulletin, section Archives: http:/ / formation-profession.org/files/495/ articles/chronique internationale.pdf

Garfinkel, H. (1967). Studies in ethnomethodology. Englewood Cliffs, NJ: Prentice Hall.

Garfinkel, H. (2001). Le programme de l'ethnométhodologie. Dans M. De Fornel, A. Ogien et L. Quéré (dir.), L'ethnométhodologie. Une sociologie radicale (p. 31-56). Paris: La Découverte de Syros.

Gauthier, C., Bissonnette, S., Richard, M. et Djibo, F. (2003). Pédagogies et écoles efficaces dans les pays développés et en développement. Document d'appui préparé pour la biennale de l'Association pour l'éducation et le développement en Afrique [AEDA]. Paris : AEDA. Récupéré le 5 février 2007 du site de l'Association, section Réunions biennales - Réunion biennale de 2003 sur la qualité - Publications Documents d'appuis - Rénovation pédagogique et développement professionnel des enseignants: http:/ / www.adeanet.org/publications biennale/fr 2003bienpubs background.html

Hans-Drouin, A.-M. (1996, octobre). La culture, une spirale de sens [version électronique]. Le Télémaque: éducation et philosophie, 7-8, p. 3-40. Récupéré le 14 février 2007 du site personnel de René Barbier: http:/ / barbier-rd.nom.fr/CultSpiraleSens.html

Houssaye, J. (1988). Le triangle pédagogique. Berne : Peter Lang.

Jessen, M. (1999). L'Afrique subsaharienne. Dans M. Tawfik, G. Bartagnon et Y. Courrier (dir.), Rapport mondial sur la communication et l'information 19992000 (p. 201-221). Paris: UNESCO. Récupéré le 14 février 2007 du site de l'UNESCO, section Communication et information: http://www.unesco. org/webworld/wcir/fr/ report.html

Jodelet, D. (1991). Les représentations sociales. Paris: Presses Universitaires de France.

Karsenti, T., Savoie-Zajc, L. et Larose, F. (2001). Les futurs enseignants confrontés aux TIC: changements dans l'attitude, la motivation et les pratiques pédagogiques [version électronique]. Éducation et francophonie, 29(1), p. 1-29. Récupéré le 5 février 2007 du site de la revue: http:/ / www.acelf.ca/c/revue/

Kom, A. (2000). La malédiction francophone. Défis culturels et condition postcoloniale en Afrique. Hambourg: Lit Verlag; Yaoundé: Clé.

Lapointe, J.-J. (1996). La conduite d'une étude de besoin en éducation et en formation: une approche systémique. Québec: Presses de l’Université du Québec.

Leborgne-Tahiri, C. (2002). Universités et nouvelles technologies en Afrique de l'Ouest francophone. Passé, présent et avenir. Dakar, Sénégal: Bureau régional pour l'éducation en Afrique (BREDA) de l'UNESCO.

Lebrun, M. (2002). Théories et méthodes pédagogiques pour enseigner et apprendre. Quelle place pour les TIC dans l'éducation? Bruxelles: De Boeck Université.

Legendre, R. (1983). L'éducation totale. Montréal: VilleMarie; Paris: Nathan. 
Linard, M. (2003). Autoformation, éthique et technologies: enjeux et paradoxes de l'autonomie. Dans B. Albero (dir.), Autoformation et enseignement supérieur (p. 241-263). Paris: Hermès Sciences/Lavoisier. Version électronique récupérée le 5 février 2007 du site e-pathie du Groupe interuniversitaire de recherche sur les usages des technologies de l'information et de la communication dans l'enseignement supérieur, section Publication - Autres travaux: http://www.epathie.org/publications.htm

Loiret, P.-J. (2005). L'Université virtuelle africaine, histoire d'une mise en scène. Mémoire de Master recherche non publié, Université de Rouen, France. Récupéré le 14 février 2007 du site de l'Observatoire sur les systèmes d'information, les réseaux et les inforoutes au Sénégal (OSIRIS): http://www.osiris.sn/ article2023.html

Marchand, L. (1998). Le changement de paradigme pour un enseignement moderne. Distances, 9(2), 7126.

Marchand, L. (2001). L'apprentissage en ligne au Canada: frein ou innovation pédagogique? Revue des sciences de l'éducation, 27(2), p. 403-419.

Marchand, L. et Loisier, J. (2003). L'université et apprentissage en ligne, menace ou opportunité. Revue des sciences de l'éducation, 29(2), p. 415-437.

Martel, A. (2002). Constructivisme et formation à distance. La transition des instructivismes aux constructivismes par les technologies de la communication au service de l'enseignement/apprentissage à distance (rapport de recherche). Montréal, Canada: Réseau d'enseignement francophone à distance du Canada (REFAD). Récupéré le 14 février 2007 du site du réseau, section Rapports de recherche-Constructivisme et formation à distance: http://www.refad.ca/ recherche/constructivisme/constructivisme.html

Ministère de l'Enseignement Supérieur du Cameroun [MES]. (2001). Loi portant orientation de l'enseignement supérieur. Yaoundé, Cameroun. Récupéré le 14 février 2007 du site du ministère, section Textes réglementaires - Lois: ftp://www.minesup.gov.cm/ Ministere/Lois/LOI_PORTANT ORIENTATION . pdf

Ministère de 1'Enseignement Supérieur du Cameroun [MES]. (2004, septembre). Rapport sur le développement de l'enseignement supérieur au Cameroun. Rapport présenté à la $47^{\mathrm{e}}$ session de la Conférence internationale de l'éducation, Genève. Récupéré le 14 février 2007 du site du Bureau international d'éducation de l'UNESCO, section Conférence internationale de l'éducation - $47^{e}$ session 2004 Rapports nationaux - Cameroon: http://www.ibe. unesco.org/International / ICE47 / French/Natreps / Nrep main.htm

Moscovici, S. (1961). La psychanalyse, son image et son public. Paris: Presses Universitaires de France.

Murphy, P., Anzalone, S., Bosch, A. et Moulton, J. (2002). Améliorer les possibilités d'apprentissage en Afrique. L'enseignement à distance et les technologies de l'information et de la communication au service de l'apprentissage (Document de travail $\mathrm{n}^{\circ}$ 31). Washington, DC: Département du développement humain de la Région Afrique, Banque Mondiale. Récupéré le 15 février 2007 du site de la Banque Mondiale, section Countries - Africa - Development Topics - Human Development - Africa Region Human Development Working Paper Series: http:/ / siteresources.worldbank.org/AFRICAEXT/ $\underline{\text { Resources/no 31.pdf }}$

Ng'ethe, N. (2003, septembre). Amélioration en matière d'innovations dans l'enseignement supérieur: les innovations dans les universités en Afrique subsaharienne. Communication présentée à la Conférence régionale de formation sur le thème Améliorer l'enseignement supérieur en Afrique subsaharienne: ce qui marche! Accra, Ghana. Récupéré le 15 février 2007 du site de la Banque Mondiale, section Countries - Africa - Development Topics Tertiary Education in Africa - A Training Conference on Tertiary Education in Africa - Conference Documents Rapport sur les innovations de l'enseignement supérieur en Afrique subsaharienne: http://siteresources. 
worldbank.org/INTAFRREGTOPTEIA/Resources / njuguna_ngethe fr.pdf

Paillé, P. (1996). Échantillonnage théorique. Dans A. Mucchielli (dir.), Dictionnaire des méthodes qualitatives en sciences humaines et sociales (p. 54-55). Paris: Armand Collin.

Paillé, P. et Mucchielli, A. (2003). L'analyse qualitative en sciences humaines et sociales. Paris: Armand Colin.

Perriault, J. (2002). L'accès au savoir en ligne. Paris : Odile Jacob.

Prayal, M.-F. et Gignac, M. (2004). Les compétences transversales acquises en situation de formation à distance (rapport de recherche). Montréal, Canada: Réseau d'enseignement francophone à distance $\mathrm{du}$ Canada (REFAD). Récupéré le 14 février 2007 du site du réseau, section Rapports de recherche-Compétences transversales en FAD: http://www.refad.ca/ recherche/comp trans fad/comp trans fad.html

Saint, W. (1999, septembre). Enseignement tertiaire à distance et technologie en Afrique subsaharienne. Washington, DC: Groupe de travail sur l'enseignement supérieur de l'Association pour le développement de l'éducation en Afrique (ADEA), Banque Mondiale. Récupéré le 15 février 2007 du site de l'association, section Publications - Catalogue des publications de l'ADEA - Groupe de travail sur l'enseignement supérieur (GTES): http:/ / www.adeanet.org/publications/wghe/tert disted fr.pdf

Samoff, J., Assié-Lumumba, N. T., Jallade, L. et Cohen, M. (1996). Analyses des programmes et priorités de l'éducation en Afrique. Un examen des études sur l'éducation en Afrique menées et commandées sur financement extérieur, 1990-1994 [n ${ }^{\circ}$ de série: ED96/WS/13 (F)]. Paris: Groupe de travail sur l'analyse sectorielle en éducation, UNESCO. Récupéré le 15 février 2007 du site de l'UNESCO, section Éducation - Reconstruction, réforme et développement des systèmes éducatifs - Publications Bibliographie des documents et publications par le Groupe de travail sur l'analyse sectorielle en éducation: http:// www.unesco.org/education/educprog/erd/ french/com/esadocf.html

Sauvé, L., Wright, A. et Saint-Pierre, C. (2004). Formation des formateurs en ligne: obstacles, rôles et compétences. La Revue internationale des technologies en pédagogie universitaire 1(2). Récupéré le 12 mars 2007 du site de la revue : http://www.profetic.org/revue

Savoie-Zajc, L. (1993). Les modèles de changement planifié en éducation. Montréal: Éditions Logiques.

Savoie-Zajc, L. (2000). La recherche qualitative/interprétative en éducation. Dans $\mathrm{T}$. Karsenti et L. Savoie-Zajc (dir.), Introduction à la recherche en éducation (p. 171-198). Sherbrooke: Éditions du CRP.

Savoie-Zajc, L. (2001). L'école renouvelée, produit de la réforme scolaire et transformations des pratiques enseignantes. Dans T. Karsenti et F. Larose (dir.), Les TIC au cœur des pédagogies universitaires (p. 69-86). Québec: Presses de l’Université du Québec.

Savoie-Zajc, L. et Dolbec, L. (1996). Quelle recherche pour quel changement? Dans J. Chevrier (dir.), La recherche en éducation comme source de changement (p. 85-101). Montréal: Éditions Logiques.

Seck, P. I. (1997). La stratégie culturelle de la France en Afrique. Paris: L'Harmattan.

Strauss, A. et Corbin, J. (1997). Basics of qualitative research: Grounded theory procedures techniques. Thousand Oaks, CA: Sage.

Tcheeko, L., Yatchou R. et Tangha, C. (2001, septembre). Outils pour le téléenseignement: expériences et précautions. Communication présentée au Colloque Initiatives 2001 : éthique et nouvelles technologies, l'appropriation des savoirs en question, tenu lors $\mathrm{du} 9^{\text {e }}$ Sommet de la Francophonie, Beyrouth, Liban. Récupéré le 12 février 2007 du site des notes du colloque, section de la Session 5: http://Www.initiatives.refer.org/ notes/session5.htm 
Trégouët, R. (1998). Des pyramides du pouvoir aux réseaux de savoirs (rapport d'information $\mathrm{n}^{\circ}$ 331). Paris : Commission des Finances, du Contrôle budgétaire et des Comptes économiques de la Nation. Récupéré le 15 février 2007 du site du Sénat français, section. Les rapports - Rapports d'information - session 1997-1998: http:/ / cubitus.senat.fr/ rapinf97.html

Valérien, J., Guidon, J., Wallet, J. et Brunswic, E. (2003). Enseignement à distance et apprentissage libre en Afrique subsaharienne. État des lieux dans les pays francophones. Paris: Groupe de travail sur l'enseignement à distance et l'apprentissage libre de l'Association pour le développement de l'éducation en Afrique (ADEA). Récupéré le 15 février 2007 du site de l'association, section Publications - Catalogue des publications de l'ADEA - Groupe de travail sur l'enseignement à distance et l'apprentissage libre : http://www.adeanet.org/publications/fr pubs wg de.html

Van der Maren, J.-M. (dir.). (1999). La recherche appliquée en pédagogie. Des modèles pour l'enseignement. Bruxelles : De Boeck Université. 


\section{Annexe 1}

Tableau 1. Avantages de l'apprentissage en ligne et contraintes associées (Lebrun, 2002; Marchand, 2001; Martel, 2002; etc.)

\begin{tabular}{|c|c|}
\hline Avantages & Contraintes \\
\hline \multicolumn{2}{|c|}{ Apprenant } \\
\hline $\begin{array}{l}\text { Élimination Odes contraintes spatiales et temporelles, } \\
\text { facilité et immédiateté de la communication }\end{array}$ & $\begin{array}{l}\text { Exigence de disposer d'un ordinateur multimédia, sentiment } \\
\text { d'isolement }\end{array}$ \\
\hline Facilitation de l'appropriation des TIC & Exigence d'habiletés techniques et sociales spécifiques \\
\hline Développement des habiletés de communication & Possible irruption de problèmes techniques imprévus \\
\hline $\begin{array}{l}\text { Accessibilité immédiate au contenu du cours, à tout } \\
\text { moment }\end{array}$ & Perte de temps lorsque le cours est mal élaboré \\
\hline Augmentation de la motivation et de la créativité & Flexibilité de la satisfaction et ambiguïté au début \\
\hline $\begin{array}{l}\text { Flexibilité de l'horaire, travail à son rythme, développe- } \\
\text { ment de l'autonomie }\end{array}$ & $\begin{array}{l}\text { Exigence de beaucoup d'efforts en temps et énergie, dispo- } \\
\text { nibilité d'un ordinateur multimédia }\end{array}$ \\
\hline $\begin{array}{l}\text { Réponse à une diversité d'attentes et de besoins } \\
\text { d'apprentissage; richesse de l'environnement en res- } \\
\text { sources informationnelles, acquisition des compétences } \\
\text { transversales }\end{array}$ & $\begin{array}{l}\text { Exigence d'habiletés d'autoformation et d'autogestion de } \\
\text { l'apprentissage; exigence d'une capacité perceptive et } \\
\text { d'analyse }\end{array}$ \\
\hline Facilitation et diversification d'interactions & $\begin{array}{l}\text { Attentes parfois peu réalistes des réactions, manque ou re- } \\
\text { tard de rétroaction }\end{array}$ \\
\hline $\begin{array}{l}\text { Multiplication d'échanges constructifs interpersonnels et } \\
\text { multidirectionnels }\end{array}$ & $\begin{array}{l}\text { Exigence d'une rapidité de synthèse d'informations et } \\
\text { d'interprétation des messages }\end{array}$ \\
\hline Facilitation du contrôle des frais de reproduction & Exigence d'un engagement financier permanent \\
\hline $\begin{array}{l}\text { Développement d'une pensée collective, globale et faci- } \\
\text { litatrice de la socialisation par la communication }\end{array}$ & Risque d'une perte de l'identité culturelle individuelle \\
\hline $\begin{array}{l}\text { Facilitation de l'accès aux experts de renommée inter- } \\
\text { nationale }\end{array}$ & Exigence de la clarté du style des communications \\
\hline \multicolumn{2}{|c|}{ Accompagnateur } \\
\hline $\begin{array}{l}\text { Disponibilité permanente du matériel didactique; modifi- } \\
\text { cation continue des contenus des cours; facilitation } \\
\text { d'interactions avec les apprenants; maintien d'une atti- } \\
\text { tude de découverte }\end{array}$ & $\begin{array}{l}\text { Exigence de la disponibilité en temps et en énergie néces- } \\
\text { saires pour entreprendre des recherches, améliorer le } \\
\text { contenu du cours et encadrer les formés, augmentation de } \\
\text { la charge de travail }\end{array}$ \\
\hline $\begin{array}{l}\text { Amélioration de la gestion du temps lors des activités } \\
\text { pédagogiques; diversifications des manières d'interagir } \\
\text { avec les apprenants }\end{array}$ & $\begin{array}{l}\text { Exigence des compétences transversales technologiques et } \\
\text { de gestion de conflits interpersonnels inattendus }\end{array}$ \\
\hline Possibilité d'enregistrer les interactions du cours & Émergence de problèmes techniques imprévus et du stress \\
\hline $\begin{array}{l}\text { Motivation et ouverture d'esprit : discernement des fonc- } \\
\text { tions et rôles, mutation conceptuelle de formation }\end{array}$ & $\begin{array}{l}\text { Peur de la menace des intérêts personnels et de l'amour- } \\
\text { propre face aux préjugés, de la perte du contrôle de sa pro- } \\
\text { fession et de la valorisation de l'investissement personnel, } \\
\text { ainsi que de la reconnaissance pour la promotion }\end{array}$ \\
\hline $\begin{array}{l}\text { Amélioration de l'image de l'institution par la modernisa- } \\
\text { tion; facilitation de la gestion administrative et financière; } \\
\text { amélioration de l'accès à la formation, réduction et maî- } \\
\text { trise des dépenses }\end{array}$ & $\begin{array}{l}\text { Engagement financier permanent pour : la formation conti- } \\
\text { nue, le soutien technique et pédagogique, l'entretien des } \\
\text { locaux équipés; gestion des droits d'auteurs et de primes, } \\
\text { etc. }\end{array}$ \\
\hline
\end{tabular}




\section{Notes}

${ }^{1}$ La saturation de la connaissance renvoie au concept d'échantillonnage théorique, qui consiste à s'entretenir avec un nombre d'interviewés croissant, jusqu'à ce que les discussions n'apportent rien de nouveau dans les données déjà constituées (Paillé, 1996; Strauss et Corbin, 1997).

${ }^{2}$ Dans la perspective constructiviste, l'enseignement est une activité intentionnelle, «dont les buts peuvent être des gains dans les connaissances, un approfondissement de la compréhension, le développement des compétences en résolution de problèmes, ou encore des changements dans la perception, les attitudes, les valeurs et les comportements» (Lebrun, 2002, p. 35). 